\section{SKELETAL MUSCLE}

\section{Work-induced Growth}

\section{from a Correspondent}

WORK-INDUCED skeletal muscle enlargement is common among athletes and physical culture enthusiasts. The mechanism by which this muscular enlargement takes place, however, is still not established, though some light is now shed on the subject by Jablecki, Heuser and Kaufman (J. Cell. Biol., 57, 743 ; 1973) who studied RNA synthesis in hypertrophying muscle of rats.

These authors confirm earlier observations (reviewed by Goldberg in Cardiac Hypertrophy (edit. by Alpert), Academic Press, 1971) that RNA synthesis is an important prerequisite to work-induced muscular growth and give evidence that the site for this RNA synthesis is connective tissue. They observed that treatment of animals with actinomycin (an inhibitor of RNA synthesis) prevented muscular hypertrophy and thus confirm that new RNA synthesis is essential. Localization of RNA synthesis was determined by autoradiography ; comparisons of light autoradiographs of control and hypertrophying muscles injected with tritiated uridine showed there to be an increased deposit of silver grains in the hypertrophied muscle. The grains were deposited almost exclusively in the connective tissue, few grains being visible on the muscle fibres themselves. Evidence that the radioactivity was associated with RNA was given by the absence of silver deposit on the autoradiograph when the animal had been pretreated with actinomycin $\mathbf{D}$.

During hypertrophy there was a concomitant increase in proliferation of connective tissue, as had been observed by Sobel and Kaufman (Archs Biochem. Biophys., 137, $469 ; 1970)$ who noticed that in the distal portion of the muscle proliferation was most extensive; they therefore used the central "less contaminated" portion of the muscle for their studies and hence failed to associate RNA synthesis with connective tissue. Jablecki et al. found a dramatic difference when comparing the rates of uptake of tritiated uridine in the distal and proximal portions of hypertrophying and control muscles: in hypertrophied muscle, the distal portion incorporated $320 \%$ more radioactivity into RNA than the control as compared with a $60 \%$ increase above the control level in the proximal portion. Conversely, preliminary experiments suggest that decreased activity of muscles, produced by spinal section, may be accompanied by a decreased rate of RNA synthesis ; however, further experiments are necessary to verify this.

The work of Jablecki et al. can be correlated with observations on hyper- trophying cardiac muscle: there is a transient increase in rate of RNA synthesis during hypertrophy; hypertrophy is also accompanied by proliferation of connective tissue. The site of RNA synthesis, however, remains to be determined. Although skeletal and heart muscles are known to be very different new ideas pertaining to the mechanism for hypertrophy in skeletal muscle should stimulate similar investigations on cardiac muscle.

\section{ECHOLOCATION}

\section{Tuning in to a Target}

from our Animal Behaviour Correspondent THE horseshoe bats (Rhinolophidae) echolocate by emitting an ultrasonic cry through the nose and beaming it by means of the nose "leaf". The cry consists of a long part of constant frequency, followed by a short frequency-modulated pulse. It seems likely that the time lapse between this f.m. terminal flourish and the f.m. part of the returning echo gives the bat information about the distance between it and its target. The long constant frequency portion, on the other hand, seems to be used to measure the relative speed of the bat and the target. As the bat flies towards a target, the echo from the cry will be higher in frequency than the cry itself because of the Doppler shift.

For a stationary target, the extent of the Doppler increase will be a function of the bat's own flight speed. It has been shown that the bat's auditory system is especially sensitive to the frequency of the bat's own cry Dopplershifted by this amount. Neurophysiological studies of the inferior colliculus of Rhinolophus have shown that this auditory part of the brain responds both when the echo begins ("on-response") and when it ends ("off-response"). The maximum on-response is obtained at a frequency of $0.5 \mathrm{kHz}$ above that of the off-response. H.-U. Schnitzler (J. comp. Physiol., 82, 79; 1973) now shows that Rhinolophus has a very sensitive feedback system which acts to keep the frequency of the emitted cries such that the returning echo is just at the best frequency for the off-response, so giving the bat high sensitivity to its own echo. $\mathrm{He}$ experimentally altered the amount of Doppler shift which a bat's cry underwent by making the animal fly in a $\mathrm{He}-\mathrm{O}_{2}$ mixture, where the speed of sound is higher than in air. The bat correctly compensated for this change, and altered the frequency of its outgoing cries so that the echoes still returned at the best frequency for the off-response.

Schnitzler suggests that by having the on-response of the evoked potentials in the inferior colliculus tuned to a slightly higher frequency than that of the offresponses, the bat can be very sensitive to unexpected movement of the target itself. If an object moves towards a bat, this will impose an additional Doppler increase on the echo, which will then fall into the frequency area of the lowest on-threshold.

This finely tuned feedback system thus not only enables the bat to hear its own echoes particularly sharply when the target is not moving (using the offresponses), but it also facilitates the detection of additional target movement (using the on-responses). How the system works with such accuracy is far from understood. The problems of such things as how the bat compares the

\title{
More About Foetal Haemoglobin
}

THE discovery by Schroeder and Huisman and their coworkers in 1968 that the $\gamma$ chain of human foetal haemoglobin (Hb-F) is heterogeneous, and that the two types of $\gamma$ chain- ${ }^{\mathbf{G}} \gamma$ and ${ }^{\mathrm{A}} \gamma$, with a glycyl or an alanyl residue at position 136 respectively-are the products of nonallelic structural genes, has made possible the study of several new features of the developmental control mechanisms for globin chain synthesis.

In 1971, the same group of workers showed that the $70: 30$ ratio of ${ }^{G} \gamma$ to ${ }^{A} \gamma$ chains found at birth decreases during the first year of life until the adult ratio of $40: 60$ is attained. Thus the repression of $\gamma$-chain synthesis and activation of $\beta$ - and $\delta$-chain synthesis coincides with a change in the relative activities of the ${ }^{\mathbf{G}} \boldsymbol{\gamma}$ and ${ }^{\mathbf{A}} \gamma$ genes. More recently, in 1972, a study of $\mathrm{Hb}-\mathrm{F}$ variants abnormal in either the ${ }^{\mathbf{G}} \gamma$ or ${ }^{\mathbf{A}} \gamma$ chain led them to the conclusion that there are in fact four non-allelic $\gamma$-chain structural gene loci $\left({ }_{m}^{\theta} \gamma,{ }_{z}^{\theta} \gamma,{ }_{m}^{A} \gamma\right.$ and ${ }_{z}^{A} \gamma$ ) with relative activities at birth of approximately $4: 2: 2: 1$. The proportions of the variants at birth indicated that $\mathbf{H b}-\mathrm{F}$ (Malta I) and $\mathrm{Hb}_{\mathrm{b}} \mathrm{F}_{\mathrm{x}}$ (Negro) were produced by mutants at the ${ }_{m}^{a} \gamma$ and ${ }_{z}^{\theta} \gamma$ loci respectively.

Further support for this striking example of gene multiplicity has now come from a study (see Huisman in Nature New Biology for July 18) of the postnatal changes in the relative properties of these two variants in heterozygotes during the first six months of life. For Hb-F (Malta I) the average proportion (relative to total $\mathrm{Hb}-\mathrm{F}$ ) declined from about $37 \%$ at birth to about $13 \%$ at 72-134 days of age. For Hb-F (Negro), by contrast, the average proportion at birth (about 11\%) showed no postnatal decrease. This difference is consistent with the view that there are indeed two ${ }^{G} \gamma$ structural loci, with separate control mechanisms operating during the $\gamma \rightarrow \beta$ switch. 